\title{
Adjuvant chemotherapy with bevacizumab (i.p.) can prolong survival time of patients with advanced ovarian cancer after cytoreduction
}

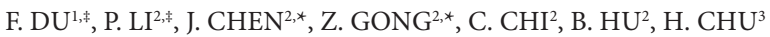 \\ ${ }^{1}$ The First clinical college of DaLian Medical University, DaLian, LiaoNing, 116000, P.R. China; ${ }^{2}$ Department of Oncology and ${ }^{3}$ The Central \\ laboratory of Yantai Yu Huangding Hospital, Affiliated Hospital of Medical College Qingdao University, Yantai, Shandong, 264000, P.R. China
}

*Correspondence: chenjianyt@163.com; gongzhaohuayt@163.com

${ }^{*}$ Contributed equally to this work.

Received June 3, 2016 / Accepted August 12, 2016

\begin{abstract}
Objective To assess whether adjuvant chemotherapy with bevacizumab intraperitoneal (i.p.) is better than adjuvant chemotherapy with bevacizumab intravenous (i.v.) or adjuvant chemotherapy only for patients with advanced ovarian cancer after cytoreduction. Selected patients with advanced ovarian cancer after cytoreduction were divided into three groups depend on their will: adjuvant chemotherapy only; adjuvant chemotherapy combined with bevacizumab intravenous (i.v.); adjuvant chemotherapy combined with bevacizumab intraperitoneal (i.p.). Patients in three groups were given 4-6 cycles of treatment and followed up for 3 years. posttreatment outcome, longterm survival and side effects were prospectively recorded and analyzed. Disease control rate (DCR) of three groups are $73.3 \%, 90.0 \%, 93.3 \%$ respectively $(\mathrm{P}<0.05)$. Remission rate (RR) and DCR of patients with bevacizumab intraperitoneal (i.p.) are better than that of patients with bevacizumab intravenous (i.v.) $(\mathrm{P}<0.05)$. The mean progression free survival (PFS) of patients with bevacizumab intraperitoneal (i.p.) (15.34 months) was longer than patients with bevacizumab intravenous (i.v.) (13.19 months), and both of two were longer than that of patients with adjuvant chemotherapy only $(9.60$ months $)\left(\chi^{2}=52.11, \mathrm{P}<0.001\right)$. The mean overall survival (OS) of patients with bevacizumab intraperitoneal (i.p.) (17.32 months) is longer than that of patients with adjuvant chemotherapy only (13.55 months) and patients with bevacizumab intravenous (i.v.) (15.17 months) $\left(\chi^{2}=75.01\right.$, $\mathrm{P}<0.001)$. Incidence of hypertension and arrhythmia of groups with bevacizumab are higher than that of patients with adjuvant chemotherapy only $(\mathrm{P}<0.05)$. Standard chemotherapy combined with bevacizumab is reliable, safe and feasible for advanced ovarian cancer after cytoreduction without severe adverse effects. Bevacizumab intraperitoneal perfusion is better than that intravenous drip.
\end{abstract}

Key words: bevacizumab, ovarian cancer, adjuvant chemotherapy

Ovarian cancer is one of the most common malignant tumor in female reproductive system. Morbidity of ovarian cancer is the third, second only to cervical cancer and uterine cancer in female reproductive system in China. Because of difficulty in early diagnosis, the prognosis of ovarian cancer patients especially for patients who missed chance of radical operation is poor. Although it is reported that 5 -year survival rate of ovarian cancer is $42.9 \%$, more than $80 \%$ of them relapsed and died within 1 year [1]. Cytoreduction and interval debulking surgery, chemotherapy, radiotherapy and targeted therapy are the main therapeutic methods for ovarian cancer now. Cytoreduction and medication are important because of difficulty in early diagnosis. Clinical trial GOG111 chaired by Gynecologic Oncology Group has revealed that progression- free survival (PFS) and overall survival (OS) of chemotherapy regimen: paclitaxel $\left(135-175 \mathrm{mg} / \mathrm{m}^{2}\right.$, i.v. $)$ on the first day and carboplatin (300-400 $\mathrm{mg} / \mathrm{m}^{2}$, i.v.) on the first day (PC) are better than cyclophosphamide combined with cisplatin [2]. Furthermore, its safety was testified by clinical trial GOG158 [3]. Except intravenous infusion (i.v.), intraperitoneal perfusion (i.p.) is a new and significant administration routes of chemotherapeutic drugs. For cancer patients after cytoreduction, OS of group cisplatin $\left(75 \mathrm{mg} / \mathrm{m}^{2}\right.$, i.p. $)$ is obviously longer than that of group cisplatin $\left(75 \mathrm{mg} / \mathrm{m}^{2}\right.$, i.v.) (66.9 months v.s 49.5 months). However, drugs toxicity is more severe in group cisplatin (75 mg/m², i.p.) [4]. Other chemotherapeutic drugs, such as bevacizumab, it is not clear yet if administration route can influence clinical efficacy. 
In this article, a prospective study was administrated in $\mathrm{Yu}$ Huangding hospital from August, 2008 to May, 2013 to explore the clinical efficacy and safety of adjuvant chemotherapy with bevacizumab (i.p.) for patients with ovarian cancer after cytoreduction.

\section{Patients and methods}

Patient selection. The study protocol was approved by the Yu Huangding hospital local ethical committee. Eligible patients presented with advanced ovarian cancer (III-IV) after cytoreduction, without cardiac, liver, renal dysfunction and other malignant tumors administrated to Yu Huangding hospital from August, 2008 to May, 2013 ( $n=90)$. Eastern Cooperative Oncology Group Test of selected patients can be less than 4 scores. Expected survival time can be more than 6 months. Patients who have been administrated PC chemotherapy before surgery were excluded from the analysis. Written informed consent was obtained before treatment.

Grouping. Patients were divided into 3 groups based on their will: patients $(n=30)$ in group 1were given chemotherapy only; patients $(\mathrm{n}=30)$ in group 2 were given chemotherapy combined with bevacizumab $(5 \mathrm{mg} / \mathrm{kg}$, iv); patients $(\mathrm{n}=30)$ in group 3 were given chemotherapy combined with bevacizumab (200 mg, i.p.).

Treatment. Every patients accepted 4-6 cycles of chemotherapy, every 21 days as one cycles. Group 1: Twelve hours and six hours before chemotherapy, dexamethasone $(10 \mathrm{mg})$ was given to prevent drug allergy. Chemotherapy regimen: paclitaxel (135 175 mg/m² , i.v., from Bristol-Myers Squibb Caribbean Company in United States) on the first day and carboplatin (300 400 mg/m², i.v., from QiluPharmacy Co., Ltd. China) on the first day. Group 2: Chemotherapy was same as that mentioned above. Bevacizumab $(5 \mathrm{mg} / \mathrm{kg}$ ) dissolved into 100 $\mathrm{ml} 0.9 \%$ sodium chloride solution was given intravenously on second day every cycle of treatment. Group 3: Chemotherapy was same as that mentioned above. Bevacizumab (200 mg) dissolved into $100 \mathrm{ml} 0.9 \%$ sodium chloride solution was given intraperitoneally on second day every cycle of treatment.

Follow-up and data collection. Starting point of follow-up is the day after treatment. End of follow-up is death or relapse or 3 years after treatment. Seven cases was lost to follow-up. Post-treatment assessment included serum CA125, computed tomographic scan of the abdomen.

Efficacy and safety evaluation. Clinical efficacy was classified into complete remission (CR), partial remission (PR), steady disease (SD), progression disease (PD) based on Response Evaluation Criteria In Solid Tumors (RECIST). Remission rate (RR) including rate of $\mathrm{CR}$ and $\mathrm{PR}$. Disease Control Rate (DCR) including rate of CR, PR, SD. Overall survival (OS) is from starting point to the end of follow-up or death. PFS is from starting point to the end of follow-up or relapse.

Side effect. Side effect of chemotherapeutic drugs is evaluated as grade I-IV depend on Common Toxicity Criteria Version 3.0 of National Cancer Institute (NCI).

Statistical analysis. Data are expressed as mean \pm standard deviation unless indicated otherwise. Differences between 3 groups of a continuous variable were assessed by Student's t test or, when data distribution was nonnormal, by the Mann-Whitney U-test. Survival time was analyzed with Kaplan-Meier method, and survival differences were checked by log-rank test. Difference is regarded as meaningful when $\mathrm{P}$ value was less than 0.05 . All data analysis is made by SPSS 21.0 software package (SPSS Inc., Chicago, IL, USA).

\section{Results}

Information of cases. Clinical characteristics, treatment details of cases in three groups are provided in Table 1. There were no significant differences between the three group regarding characteristics $(\mathrm{P}>0.05)$.

Table 1. Clinical characteristics of patients.

\begin{tabular}{|c|c|c|c|c|}
\hline Group & $1(\mathrm{n}=30)$ & $2(\mathrm{n}=30)$ & $3(\mathrm{n}=30)$ & P-Value \\
\hline Age, $y$ & $54.24 \pm 8.61$ & $59.23 \pm 5.42$ & $57.38 \pm 13.14$ & - \\
\hline \multicolumn{5}{|l|}{ Histological subtypes n (\%) } \\
\hline Serous adenocarcinoma & $26(86.7)$ & $25(85.6)$ & $26(86.7)$ & 0.88 \\
\hline Mucinous adenocarcinoma & $2(6.7)$ & $4(13.3)$ & $3(10.0)$ & \\
\hline Endometrioid carcinoma & $2(6.7)$ & $1(3.3)$ & $1(3.3)$ & \\
\hline \multicolumn{5}{|l|}{ Surgical stage n (\%) } \\
\hline IIIA & $7(23.3)$ & $5(16.7)$ & $6(20.0)$ & 0.98 \\
\hline IIIB & $7(23.3)$ & $6(20.0)$ & $7(23.3)$ & \\
\hline IIIC & $14(46.7)$ & $17(56.7)$ & $16(53.3)$ & \\
\hline IV & $2(6.7)$ & $2(6.7)$ & $1(3.3)$ & \\
\hline \multicolumn{5}{|l|}{ ECOG score n (\%) } \\
\hline$<3$ & $27(90.0)$ & $29(96.7)$ & $27(90.0)$ & 0.54 \\
\hline$\geq 3$ & $3(10.0)$ & $1(3.3)$ & $3(10.0)$ & \\
\hline Cycles of treatment & $4.24 \pm 1.61$ & $4.54 \pm 0.91$ & $4.27 \pm 1.63$ & - \\
\hline
\end{tabular}




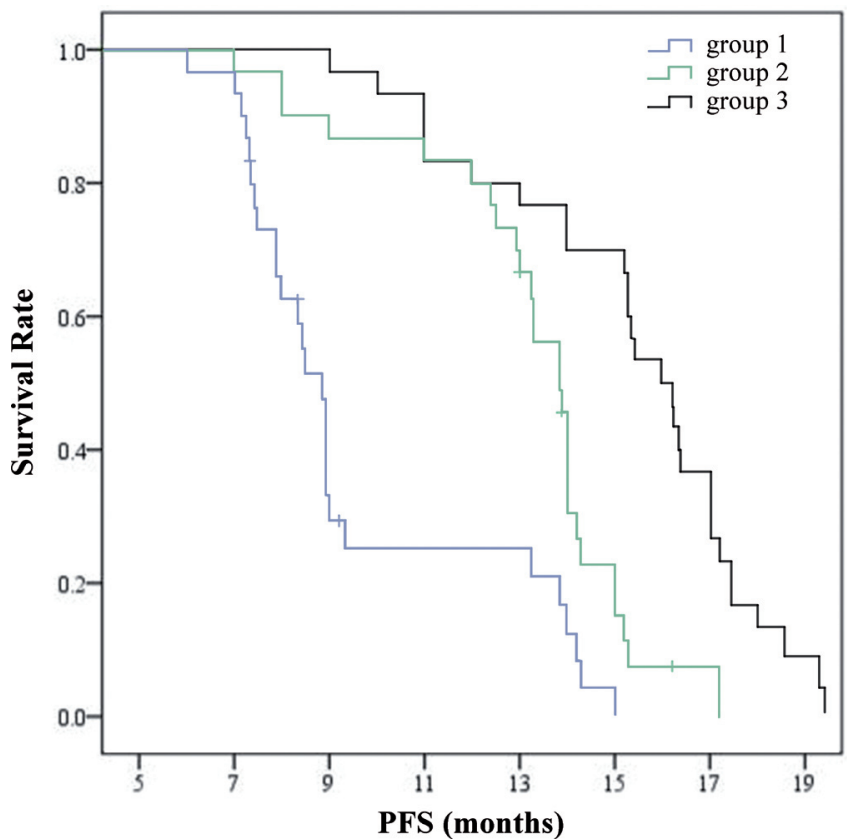

Figure 1. Progression free survival curves of three groups.

Group 1: chemotherapy only, group 2: chemotherapy combined with bevacizumab (5 mg/kg, i.v.), group 3: chemotherapy combined with bevacizumab (200 $\mathrm{mg}$, i.p.).

Data excluded from each group was represented by censored lines.

Clinical efficiency (Table 2). Rate of CR, PR of group 2 $(13.3 \%, 46.7 \%)$, group $3(13.3 \%, 50.0 \%)$ are significantly higher that of group $1(3.3 \%, 36.7 \%)$. And rate of $\mathrm{PD}$ of group 2 $(10.0 \%)$, group $3(6.7 \%)$ are obviously lower than group 1 (26.7\%). However, rate of SD among three groups are similar. RR (remission rate) of group $3(63.3 \%)$ and group $2(60.0 \%)$ are higher than that of group $1(40.0 \%)(\mathrm{P}<0.05)$. DCR of three groups are $73.3 \%, 90.0 \%, 93.3 \%$ respectively $(\mathrm{P}<0.05)$. $\mathrm{RR}$ and DCR of group 3 are better than group $2(\mathrm{P}<0.05)$.

Comparison of PFS and OS among three groups. The mean PFS of group 3 (15.34 months) was longer than group 2 (13.19 months), and both of two were longer than that of group 1 (9.60 months $)\left(\chi^{2}=52.11, \mathrm{P}<0.001\right)$. Confidence inter-

Table 2. Clinical efficiency after treatment of three groups.

\begin{tabular}{lcccc}
\hline group & & $1(\mathrm{n}=30)$ & $2(\mathrm{n}=30)$ & $3(\mathrm{n}=30)$ \\
\hline & CR & $1(3.3)$ & $4(13.3)^{\star}$ & $4(13.3)^{\star}$ \\
Clinical efficiency & PR & $11(36.7)$ & $14(46.7)^{\star}$ & $15(50.0)^{\star}$ \\
$\mathrm{n}=30, \mathrm{n}(\%)$ & SD & $10(33.3)$ & $9(30.0)$ & $9(30.0)$ \\
& PD & $8(26.7)$ & $3(10.0)^{\star}$ & $2(6.7)^{\star}$ \\
RR (\%) & & 40.0 & $60.0^{*}$ & $63.3^{* *}$ \\
DCR (\%) & & 73.3 & $90.0^{*}$ & $93.3^{* *}$ \\
\hline
\end{tabular}

${ }^{*}$ the difference is significant compare with group $1(\mathrm{P}<0.05)$, "the difference is significant compare with group $2(\mathrm{P}<0.05)$; $\mathrm{CR}$, complete remission; $\mathrm{PR}$, partial remission; $\mathrm{SD}$, steady disease; $\mathrm{PD}$, progression disease; $\mathrm{RR}$, remission rate; $\mathrm{DCR}$, disease control rate

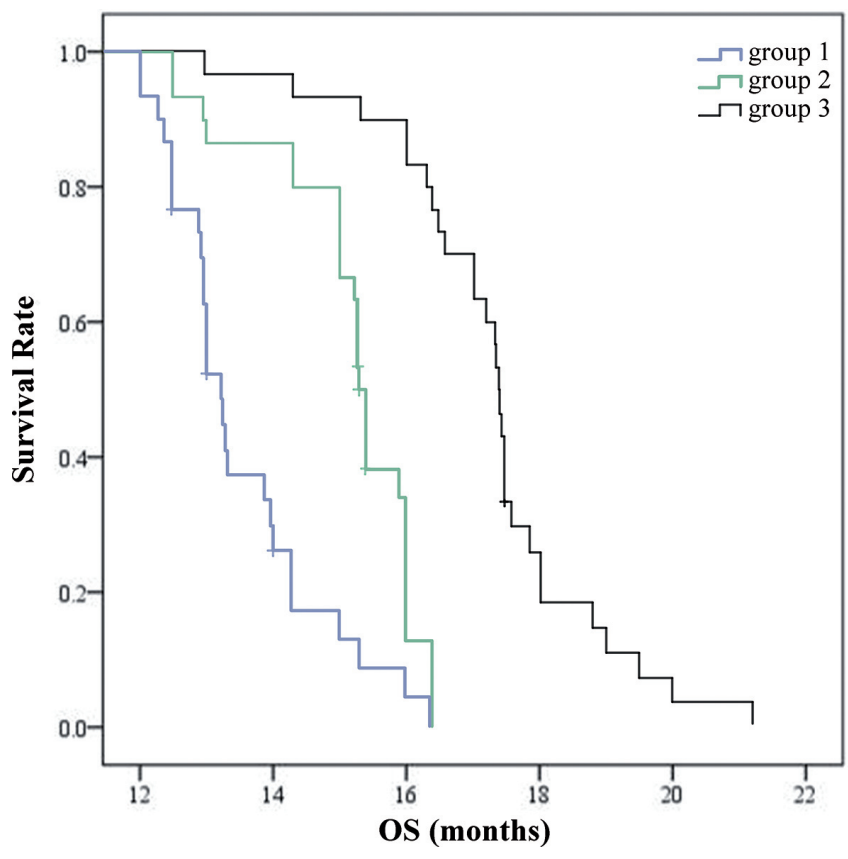

Figure 2. Overall survival curves of three groups.

Group 1: chemotherapy only, group 2: chemotherapy combined with bevacizumab ( $5 \mathrm{mg} / \mathrm{kg}$, i.v.), group 3: chemotherapy combined with bevacizumab (200 $\mathrm{mg}$, i.p.).

Data excluded from each group was represented by censored lines.

val (CI) in 95\% of PFS of patients in group 1 was $8.56 \pm 10.64$ months, that group 2 was $12.30 \pm 14.09$ months, that of group 3 was $14.32 \pm 16.36$ months. The mean OS of group 3 (17.32 months) is longer than that of group 1 (13.546 months) and group 2 ( 15.17 months $)\left(\chi^{2}=75.01, \mathrm{P}<0.001\right) .95 \%$ CI of OS of three groups were $13.10 \pm 13.99$ months, $14.76 \pm 15.57$ months, $16.74 \pm 17.91$ months respectively. Survival curves of PFS and OS of three groups were shown in Figure 1 and Figure 2.

Comparison of adverse effects. The incidence of side effects including myelosuppression, nausea, vomiting, diarrhoea are similar among three groups $(\mathrm{P}>0.05)$. However, incidence of hypertension and cardiotoxicity of group 2, 3 are obviously higher than that of group $1(\mathrm{P}<0.05)$. However, grade IV hypertension is few. The difference in incidence of hypertension and arrhythmia between group 2 and group 3 have no statistical significance $(\mathrm{P}>0.05)$. More importantly, arrhythmia is present only in groups with bevacizumab.

\section{Discussion}

Reportedly, approximately 225000 new cases of ovarian cancer are diagnosed worldwide, and about 140,200 women die of the disease each year [5]. Ovarian cancer, compared to other solid tumors, has a strong proclivity for early peritoneal dissemination and a high percentage of women present with advanced stage disease. Surgical cytoreduction and standard chemotherapy comprises the primary therapy. Although it is 
Table 3. Comparison of adverse effects among three groups.

\begin{tabular}{|c|c|c|c|c|c|c|}
\hline Side-effect & Grade & $1 \mathrm{n}(\%)$ & 2 n (\%) & $3 \mathrm{n}(\%)$ & $\chi^{2}$ & P-Value \\
\hline \multirow{2}{*}{ Myelosuppression } & I-II & $16(53.3)$ & $17(53.3)$ & $18(60.0)$ & 0.48 & 0.79 \\
\hline & III-IV & $2(6.7)$ & $3(10.0)$ & $1(3.3)$ & & \\
\hline \multirow{2}{*}{ Nausea, vomiting } & I-II & $18(60.0)$ & $20(66.7)$ & $19(63.3)$ & 0.10 & 0.96 \\
\hline & III-IV & $3(10.0)$ & $4(13.3)$ & $3(10.0)$ & & \\
\hline \multirow{2}{*}{ Diarrhoea } & I-II & $9(30.0)$ & $11(36.7)$ & $12(40.0)$ & 0.53 & 0.77 \\
\hline & III-IV & $5(16.7)$ & $6(20.0)$ & $4(13.3)$ & & \\
\hline \multirow{3}{*}{ Hypertension } & I & $1(3.3)$ & $14(46.7)$ & $6(20.0)$ & 8.05 & $0.02^{\#}$ \\
\hline & II & $2(6.7)$ & $3(10.0)$ & $14(46.7)$ & & \\
\hline & III & 0 & $1(3.3)$ & $1(3.3)$ & & \\
\hline \multirow{2}{*}{ Arrhythmia } & I-II & 0 & 8 & 11 & - & - \\
\hline & III-IV & 0 & 0 & 1 & & \\
\hline
\end{tabular}

\#The difference has statistical significance

reported that 5 -year survival rate of ovarian cancer is $42.9 \%$, more than $80 \%$ of them relapsed and died within 1 year with a median time to first recurrence of 16 months [6].

Recently, bevacizumab has become a hot topic because of its significant clinical efficiency in advanced ovarian cancer. Bevacizumab is a monoclonal anti-vascular endothelial growth factor (VEGF)-A antibody targeting tumor angiogenesis. As is known to all, tumor angiogenesis plays a pivotal role in the growth and metastasis of cancer. When tumor become larger than 1-2 $\mathrm{mm}$ in size, it must recruit a blood supply from surrounding host tissue [7]. An angiogenic switch, which is associated with an increased growth and metastatic potential, was activated by the activation of tumor oncogenes, tissue hypoxia, and increased tumor expression of multiple proangiogenic factors, including VEGF, fibroblast growth factor (FGF), platelet-derived growth factor (PDGF), angiopoietins (Ang1 and Ang2), and others [8]. VEGF-A is one of a family of seven soluble VEGF ligands, firstly found from hepatocarcinoma cells of guinea pig by Senger in 1983 [9]. VEGF-A preferentially binds VEGFR-1 and VEGFR-2, which are the most important receptors for VEGF-A- mediated angiogenesis [10]. It is the reason why bevacizumab demonstrated significant anticancer efficiency that bevacizumab can competitively binds VEGF-A and inhabits angiogenesis.

In ovarian cancers, VEGF is detectable by immunohistochemistry in ovarian tumors, ascites and serum sample. Moreover, its expression levels is cancer specific, with high levels of expression only in invasive cancers $[11,12]$. VEGF expression also has closed relation with ovarian cancer stage and prognosis [13-15]. Therefore, numbers clinical clinical studies about clinical efficiency of bevacizumab for ovarian cancer have been carried out. About 218, 1,873 women with stage III or IV previously untreated or incompletely resected ovarian cancer were recruited into Gynecologic Oncology Group (GOG). The treatment agents of control group carboplatin, paclitaxel and placebo. The experimental group was given bevacizumab $(15 \mathrm{mg} / \mathrm{kg})$ and followed by bevacizumab maintenance. Experimental group resulted in an improved PFS (median PFS 14.1 months vs 10.3 months $(\mathrm{P}=0.001)$. But there was no difference in OS. In our study, the mean PFS of group 3 and group 2 were longer than that of group $1(15.34,13.19$ vs 9.60$)\left(\chi^{2}=52.11, \mathrm{p}<0.001\right) .95 \% \mathrm{CI}$ (confidence interval) of PFS of patients in group 1 was 8.56 \pm 10.64 months, that group 2 was $12.30 \pm 14.09$ months, that of group 3 was $14.32 \pm 16.36$ months. The mean OS of two groups with bevacizumab are longer than that of group 1 . $95 \%$ CI of OS of three groups were $13.10 \pm 13.99$ months, 14.76 \pm 15.57 months, $16.74 \pm 17.91$ months respectively. The results of PFS are similar with that of GOG. It tells us the significant anticancer efficacy of bevacizumab. The results of OS in our study have something difference with that reported before because of limited number of cases recruited, postoperative cases in all and different dose of bevacizumab. Unexpectedly, in our study, results that bevacizumab (i.p.) obtain a better efficient in PFS, OS and RR, DCR than that of bevacizumab (i.v.). In 1970, the American Cancer Society first promoted the concept of intracavitary chemotherapy that could maintain an effective drug concentration for a longer period [16]. Cisplatin, oxaliplatin, carboplatin are the primary clinical chemotherapeutic drugs for thoracic and abdominal cavity perfusion and has demonstrated better results than given intravenous drip [17]. The authors tend to believe the reason why intraperitoneal perfusion can improve the efficacy of bevacizumab may be that an optimal concentration is maintained for a longer time.

Bevacizumab is well accepted and tolerated. The toxicities most attributed to bevacizumab include GI perforation, hypertension, proteinuria, venous thromboembolism (VTE), impaired wound healing. Hypertension is the most common side effect of bevacizumab. The rate of grade 3 hypertension varies from $2 \%$ to $23 \%$ in ovarian cancer studies [18]. And grade 2 hypertension occurs in $20 \%$ of patients in the AURELIA trial [19]. In our study, side effects such as myelosuppression, nausea, vomiting, diarrhea are similar among three groups because of result from standard chemotherapy. However, rate of hypertension in groups with bevacizumab 
is higher than group 1, with grade 3 occurring in $3.3 \%$ of patients. It is in accordance with reported before. No case was withdrawed because of hypertension. But four cases in groups with bevacizumab were given oral antihypertensive agents and improved. Cardiotoxicity is a rare side effect of bevacizumab, with no exact occurrence rate. However, 20 cases in groups with bevacizumab suffered from arrhythmia and one of them accepted treatment of oral agents. Moreover, the rate of arrhythmia in group 3 is higher than that of group 2. Only two of them have history of arrhythmia. Some researcher believe that cardiotoxicity of bevacizumab may be related with "target effect", inhibition VEGF that is indispensable for cardiac function, and "missing target effect", targeted to other important molecular site with significant function for heart when primary targeting site changes [20]. So, cardiotoxicity of bevacizumab has closed relation with regulation of vascular contraction and maintain blood pressure of VEGF. VEGF can induce diastole of endothelium dependent coronary artery by stimulate endothelial cells release Nitrous oxide (NO) and Prostacyclin (PEI2) and then induce decrease of hypertension. Izumiya $Y$ et al also proved that VEGF can reduce cardiac hypertrophy caused by overloaded [21]. Therefore, bevacizumab, inhibition of VEGF, can lead to vasoconstriction and hypertension. In our study, all cases suffered from arrhythmia were reversed by themselves except one by oral agents. The reason why occurrence of arrhythmia is higher in group 3 is higher than that of group 2 may be that optimal concentration of bevacizumab can be obtain in short time and maintain for a long time.

In conclusion, standard chemotherapy combined with bevacizumab is reliable, safe and feasible for advanced ovarian cancer after cytoreduction. Moreover, bevacizumab intraperitoneal perfusion is better than that intravenous drip. However, arrhythmia may be a sever side effect of bevacizumab, and may be worsen with increased concentration. More clinical investigations are still required regarding the required dosage of bevacizumab intraperitoneal perfusion.

Acknowledgments: This work was supported by National Natural Science Foundation of China (81071758); Shandong Science and Technology Development Project (2015GSF118142); Natural Science Foundation of Shandong Province Joint Programme (ZR2015HL069); and Yantai Yu Huangding Hospital Initiative Foundation for Young Scientist (201402); Yantai Science and Technology Program (2015WS018).

\section{References}

[1] WEBB PM, DE FAZIO A, PROTANI MM, IBIEBELE TI, NAGLE CM et al. Circulating 25-hydroxyvitamin D and survival in women with ovarian cancer. Am J Clin Nutr 2015; 102: 109-114. https:/doi.org/10.3945/ajcn.114.102681

[2] MCGUIRE WP, HOSKINS WJ, BRADY MF, KUCERA PR, PARTRIDGE EE et al. Cyclophosphamide and cisplatin compared with paclitaxel and cisplatin in patients with stage III and stage IV ovarian cancer. N Engl J Med 1996; 334: 1-6. https:/doi.org/10.1056/NEJM199601043340101
[3] OZOLS RF, BUNDY BN, GREER BE, FOWLER JM, CLARKEPEARSON D et al. Phase III trial of carboplatin and paclitaxel compared with carboplatin and paclitaxel in patients with optimally resected stage III overian cancer: A gynecologic oncology group study. J Clin Oncol 2003; 21:3194-3200. https:/ doi.org/10.1200/JCO.2003.02.153

[4] MARKMAN M, BUNDY B, ALBERTS D, FOWLER JM, CLARK-PEARSON DL et al. Phase III trial of standard dose intravenous cisplatin plus paclitaxel versus moderately high-dose carboplatin followed by intravenous paclitaxel and inraperitoneal cisplatin in small-volume stage III overian carcinoma: an intergroup study of the Gynecologic Oncology Group,Southwestern Oncology Group, and Eastern Cooperative Oncology Group. J Clin Oncol 2001; 19: 1001-1007.

[5] BEREK JS, CRUM C, FRIEDLANDER M. Cancer of the ovary, fallopian tube, and peritoneum. Int J Gynaecol Obstet 2012; 119 : S118-S129. https:/doi.org/10.1016/S0020-7292(12)60025-3

[6] GIMBRONE MA, LEAPMAN SB, COTRAN RS, FOLKMAN J. Tumor dormancy in vivo by prevention of neovascularization. J Exp Med 1972; 136: 261-276. https:/doi.org/10.1084/ jem.136.2.261

[7] HANAHAN D, FOLKMAN J. Patterns and emerging mechanisms of the angiogenic switch during tumorigenesis. Cell 1996; 86: 353-364. https:/doi.org/10.1016/S0092-8674(00)80108-7

[8] SENGER D, GALLIS J, DVORAK AM. Tumor cells secrete a novel heparin-binding growth factor specific for vascular endothelial cells. Biophys Res Commum 1989; 161: 851-858. https:/doi.org/10.1016/0006-291X(89)92678-8

[9] GERBER HP, FERRARA N. Pharmacology and pharmacodynamics of bevacizumab as monotherapy or in combination with cytotoxic therapy in preclinical studies. Cancer Res 2005; 65: 671-680.

[10] ABU-JAWDEH GM, FAIX JD, NILOFF J, TOGNAZZI K, MANSEAU E et al. Strong expression of vascular permeability factor (vascular endothelial growth factor) and its receptors in ovarian borderline and malignant neoplasms. Lab Invest 1996; 74: 1105-1115.

[11] YAMAMOTO S, KONISHI I, MANDAI M, CUSCHIERI K, LYMAN R et al. Expression of vascular endothelial growth factor (VEGF) in epithelial ovarian neoplasms: correlation with clinicopathology and patient survival, and analysis of serum VEGF levels. Br J Cancer 1997; 76: 1221-1227. https:/ doi.org/10.1038/bjc.1997.537

[12] PALEY PJ, STASKUS KA, GEBHARD K, MOHANRAJ D, TWIGGS LB et al. Vascular endothelial growth factor expression in early stage ovarian carcinoma. Cancer 1997; 80: 98-106. https:/doi.org/10.1002/(SICI)1097-0142(19970701)80:1<98::AID-CNCR13>3.0.CO;2-A

[13] SHEN G, GHAZIZADEH M, KAWANAMI O, SHIMIZU $\mathrm{H}$, JIN E et al. Prognostic significance of vascular endothelial growth factor expression in human ovarian carcinoma. Br J Cancer 2000; 83: 196-203.

[14] LI L, WANG L, ZHANG W, TANG B, ZHANG J et al. Correlation of serum VEGF levels with clinical stage, therapy efficacy, tumor metastasis and patient survival in ovarian cancer. Anticancer Res 2004; 24: 1973-1979. 
[15] BURGER RA, BRADY MF, BOOKMAN MA, FLEMING GF, MONK BJ et al. Incorporation of bevacizumab in the primary treatment of ovarian cancer. N Engl J Med 2011; 365 : 2473-2483. https:/doi.org/10.1056/NEJMoa1104390

[16] CARMIGNANI L, BIANCHI R, COZZI G, GRASSO A, MACCHIONE $\mathrm{N}$ et al. Intracavitary immunotherapy and chemotherapy for upper urinary tract cancer: current evidence. Rev Urol 2013; 15: 145-153.

[17] JIANG L, LI P, GONG Z, HU B, MA J et al. Effective Treatment for Malignant Pleural Effusion and Ascites with Combined Therapy of Bevacizumab and Cisplatin. Anticancer Res 2016; 36: 1313-1318.

[18] RANDALL LM, MONK BJ. Bevacizumab toxicities and their management in ovarian cancer. Gynecol Oncol 2010; 117: 497-504. https:/doi.org/10.1016/j.ygyno.2010.02.021
[19] PUJADE-LAURAINE E, HILPERT F, WEBER B, HU B, MA J et al. Bevacizumab combinedwith chemotherapy for platinum-resistant recurrent ovarian cancer: The AURELIA open-label randomized phase III trial. J Clin Oncol 2014; 32: 1302-1308. https:/doi.org/10.1200/JCO.2013.51.4489

[20] CHENG H, FORCE T. Why do kinase inhibitors cause cardiotoxicity and what can be done about it. Prog Cardiovasc Dis 2010; 53: 114-120. https:/doi.org/10.1016/j. pcad.2010.06.006

[21] IZUMIYA Y, SHIOJIMA I, SATO K, SAWYER DB, COLUCCI WS et al. Vascular endothelial growth factor blockade promotes the transition from compensatory cardiac hypertrophy to failure in response to pressure overload. Hypertension 2006; 47: 887-893. https:/doi.org/10.1161/01. HYP.0000215207.54689.31 